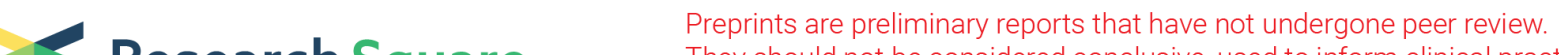 Research Square They should not be considered conclusive, used to inform clinical practice, or referenced by the media as validated information.
}

\section{Molecular Analysis of Targeted Insecticide Resistance Gene Mutations in field Aedes Aegypti and Culex Quinquefasciatus Mosquito Vectors of Arboviruses from Saudi Arabia}

\section{Yuan Fang}

National Institute of Parasite Disease, Chinese Center for Disease Control and Prevention

\section{Tambo Ernest}

Municipality of Jeddah Governorate

Jin-Bo Xue

National Institute of Parasitic Diseases, China CDC

Yi Zhang ( $\sim$ zhangyi@nipd.chinacdc.cn )

Hany A. Kamal

Ain Shams University

Fahd S. Alzahrani

Municipality of Jeddah Governorate

Mohamed H. Alzahrani

Municipality of Jeddah Governorate

\section{Xiao-Nong Zhou}

National Institute of Parasitic Diseases, China CDC

Emad I.M. Khater

Ain Shams University Faculty of Women for Arts Science and Education

\section{Research}

Keywords: acetylcholinesterase 1 gene, dengue, knockdown resistance gene, voltage gated sodium channel gene

Posted Date: May 12th, 2020

DOI: https://doi.org/10.21203/rs.3.rs-28170/v1

License: @ (i) This work is licensed under a Creative Commons Attribution 4.0 International License. Read Full License 


\section{Abstract}

Background In absence of effective and safe vaccines or drugs against mosquito-borne arboviral diseases (such as dengue, Zika and Rift Valley and West Nile), there have been an increasing pattern of insecticide use against the mosquito vectors of these diseases in integrated vector management (IVM) programs in Saudi Arabia. However, the efficacy of IVM programmes is threatened by the development of insecticide resistance in vectors. Gene mutations on target sites can be a valuable reference to the status of insecticide resistance. Jeddah, a global commercial and major port-of-entry city, is bearing the most ( $>70 \%$ ) dengue disease burden and the population of the dengue vector Aedes aegypti in Saudi Arabia. Culex quinquefasciatus is a second example as one of the major arboviral vectors in the region and a proven vector of Rift Valley fever and wide-spread in Jeddah and the rest of the western region of Saudi Arabia. However, the status of insecticide resistance and targeted site mutations on the responsible genes are not fully characterized.

Methods We randomly sampled both mosquito species, Ae. aegypti and Cx. quinquefasciatus across Jeddah by daily mosquito surveillance in 2016 to detect the resistance-associated target site mutations on the voltage gated sodium channel (VGSC) and acetylcholinesterase 1 (ace-1) genes.

Results Our findings showed that Ae. aegypti resistance-associated VGSC gene mutations revealed polymorphic mutations on the 989 (allele frequency $=65.91 \%$ ), 1016 (allele frequency $=65.91 \%$ ), and 1534 (allele frequency = $52.27 \%$ ) sites. Additionally, we documented two types of introns between exons 20 and 21, however, the I1011M point mutation was undetected. Linkage disequilibrium associations were shown between V1016G with S989P, V1016G with F1534, and V1016G with type A intron. Furthermore, no mutation on ace-1 was identified in Ae. aegypti. In CX. quinquefasciatus, homozygous L1014F/L1014F (95.23\%) on the VGSC and heterozygous G119/G119S (100\%) on ace-1 were widely distributed in the samples studied.

Conclusions To the best of our knowledge, this is the first record of the intron types between exons 20 and 21 on VGSC of Ae. aegyptipopulations, and the first report of insecticide resistance gene mutation being present in field caught $C x$. quinquefasciatus in Jeddah, Saudi Arabia. High prevalence of insecticide resistance gene mutations in local primary mosquito vector species, implies that the local Ae. aegypti mosquitoes are resistant to both pyrethroid and DDT, while the resistance to all four insecticide groups may possessed in $C x$. quinquefasciatus populations. Our findings alert the urgent need to carry out a comprehensive insecticide resistance gene mutation surveillance and monitoring to guide sustained and effective IVM planning and innovative guidelines in local predominate mosquito populations and mosquito-borne disease control and elimination in Saudi Arabia.

\section{Introduction}

Mosquitoes-borne diseases, including dengue, malaria, West Nile, Japanese encephalitis, Rift Valley fever, Chikungunya, Zika, and other emerging arboviral diseases are prevalent and are enormous threat to public health worldwide. Intensive international trade and travel, influenced by global economic integration, accelerated the spread of mosquito-borne viruses, resulting in occasional disease outbreaks even in previously endemic or disease-free areas. Since no protective vaccines and effective drugs are available for majority of these infectious diseases, the strategies on reducing the risk of transmission is mainly focused on mosquito vector control. Vector control has been relying heavily on insecticides application to reduce mosquito larval and adult 
populations in their natural habitats as well as personal protection using anti-mosquito repellents [1]. Selection pressure on insecticide target sites of mosquito vectors, resulting in the occurrence and developing of single or multiple-site mutations in the responsible genes have been reported and subsequently detected globally in major mosquito vector species belonging to the genera Anopheles, Aedes and Culex in the last few decades [2,3]. Increased insecticide target site insensitivity due to gene point mutations, and the increased or elevated activity of insecticide detoxifying enzymes are two of the major mechanisms evolved and developed by mosquito vectors for surviving the lethal effect of insecticides $[4,5]$.

At least four vector-borne viruses, including dengue virus (DENV), Alkhurma hemorrhagic fever virus, Rift Valley fever virus, and Crimean-Congo hemorrhagic fever virus have been circulating in Saudi Arabia [6]. The most important of them is dengue, with most of the disease burden and severity, mainly prevalent in the western and southwestern regions in Jeddah and Makkah, of Saudi Arabia [7-9]. A total of 5,345 confirmed dengue cases were reported in 2018 , with incidence rate of $16 / 100,000$ population, with most of cases $(92.46 \%, n=4,942)$ in Jeddah and 3.05\% $(n=163)$ in Makkah, according to the Ministry of Health of Saudi Arabia recent reports [10]. The incidence among Non-Saudi was $32.8 / 100,000$ population (most of them are expatriates from dengueendemic countries in Asia and Africa, while reached 5.8/100,000 population among Saudi citizens. Saudi Arabia was once a dengue-free country till the first dengue outbreak in Jeddah in 1994 [11]. Now all the four DENV serotypes (DENV1-4) have been reported in Jeddah and Makkah $[6,8,12]$. Jeddah is the financial and diplomatic center in Saudi Arabia, and is the major point-of-entry to the Holy city of Makkah (only $80 \mathrm{~km} \mathrm{SE}$ of Jeddah) for millions of visitors of perennial religious rituals for pilgrimage (Hajj) and Umrah as well as workers and other visitors. Aedes aegypti, the major mosquito vector of dengue, chikungunya and Zika, and $C X$. quinquefasciatus, vector of West Nile, Rift Valley fever and Japanese encephalitis, are two dominant mosquitoes in Jeddah $[13,14]$. Although there have been no reports of West Nile virus outbreaks in Jeddah, it has been reported in countries neighbouring Saudi Arabia, such as Pakistan, Iran, Egypt and Israel [15-17]. Since the distribution of the two vectors Ae. aegypti and $C x$. quinquefasciatus are widespread and both are domestic and endophagic species, there is an ever-present risk of arboviral disease outbreaks, as is the case of dengue in the last three decades, especially in those fast-growing cities with megaprojects of development and urbanization. Meanwhile, the two species suffered from extensive and long-time insecticide exposure for routine mosquito control operations using larvicides spraying of their natural breeding habitats and indoor residual spraying (IRS) using adulticides, resulting in developing of insecticide resistance, which subsequently reduced the efficacy of insecticide treatments $[7,9,11,16]$.

Aedes aegypti populations from Jeddah and Makkah showed high resistance to permethrin, deltamethrin, bendiocarb, and were tolerant to fenitrothion, based on conventional insecticide bioassays performed on adult mosquito samples collected in 2015 [18]. Noteworthy, in a ten-year period, the mortalities to pyrethroids decreased from $75-93 \%$ in 2008 to $<20 \%$ in 2017 in the Ae. aegypti population in Makkah [18]. In addition, the Jazan population was only sensitive to cyfluthrin, tolerant to deltamethrin, permethrin, fenitrothion, and resistant to Lambda-cyhalothrin, DDT, and bendiocarb [19]. It should be noted that pyrethroid group (mainly deltamethrin, cypermethrin, and cyfluthrin), sporadically the organophosphates pirimiphos-methyl and diazinon, have been the most common insecticides used in different commercial formulations over the last 20 years in the Kingdom. Whereas, resistance to DDT, permethrin and deltamethrin in $C_{x}$. quinquefasciatus from Jeddah developed as early as 1984 [20]. However, to our knowledge, no relevant record reported on insecticide resistance in $C x$. quinquefasciatus from Saudi Arabia after then, especially in field-caught individuals. 
The most prevalent insecticide resistance gene mutation is the knockdown resistance $(k d r)$ mutation on the para voltage sodium channel gene (VGSC). The mutation sites on the amino acid 1014 position on VGSC IIS for both Anopheles and Culex, and 1016 position for Aedes, were well confirmed to reduce the mosquito sensitivity to pyrethroid, and the organochlorine DDT due to cross-resistance effect [21-23]. In specific, several point mutation sites on VGSC are speculated to get involved in pyrethroid and DDT resistance in Ae. aegypti, including G923V, L982W, S989P, M1011I/G, V1016G, F1269C in domain II, and T1520I, F1552C, F1534C, D1763Y in domain III of VGSC [1, 24-26]. Among them, two point mutations, V1016G and F1534C that confer resistance to pyrethroids and DDT, are the most widespread [27-32]. Four non-synonymous (A99S, L1014F/S/C, V1016G, W1594R) and six synonymous, (L884L, G923G, A1262A, D1266D, P1270P, G1754G) mutations were found to be linked with the levels of permethrin resistance in $C x$. quinquefasciatus populations [33-35]. In addition, the replacement of the amino glycine (Gly, G) with serine (Ser, S) on position 119 of acetylcholinesterase 1 (ace-1) was found to be associated with mosquito resistance to organophosphate and carbamate insecticide groups [36]. The G119S mutation was commonly detected in Anopheles and Culex, while seldomly recorded in Aedes species [1]. In Saudi Arabia, S989P, V1016G and F1534C mutations on VGSC were reported in Ae. aegypti samples collected from Jeddah and Makkah in 2015 [18]. It is the first on insecticide resistance gene detection in Ae. aegypti populations from Saudi Arabia.

However, little is documented on the trend and impact of insecticides application in integrated vector management (IVM) programs implementation nearly for 20 years (1995-2016) on vector-borne disease threats and epidemics prevention and control in the Kingdom of Saudi Arabia $[16,17]$.

Additionally, point mutations in the VGSC gene of Ae. aegypti populations in Jeddah has not been fully characterized, especially the types of introns between exons 20 and 21, as well as whether the ace- 1 mutations in Jeddah Ae. aegyptipopulations. In Cx. quinquefasciatus, the V1016G mutation was detected in the JPP-B strain, originated from Jeddah and selected by permethrin for 20 consecutive generations [20, 34]. This mutation has not been reported in other populations of Cx. pipiens complex [37]. To the best of our knowledge since the first studies performed in the mid of 1980s, no study on insecticide resistance gene(s) in Cx. quinquefasciatus populations have been reported in the so far in Saudi Arabia. Thus, currently, there are limited information on insecticide resistance gene mutations in $C x$. quinquefasciatus against chemical insecticides in Jeddah. For the bioassay test requires numerous live mosquitoes and time-consuming [38]. Early detection and characterization of target mutations of insecticide resistance genes, as potentially valuable molecular markers, can provide a reference to estimate the local insecticide resistance level for monitoring, planning and evaluation of vector control strategy $[1,39,40]$.

This study aimed to determine the status of insecticide resistance in Ae. aegypti and Cx. quinquefasciatus mosquito vectors, by mapping the point mutations associated with $k d r$ mutations and the phylogeny of the introns between the exons 20 and 21 on VGSC, and ace-1. The results of our study will be very important for the early-detection of resistance development and in guiding IVM and insecticide application policies for the prevention and control of mosquito-borne diseases in Saudi Arabia.

\section{Materials And Methods}

\section{Mosquito Collection}


A total of 25 Ae. aegypti and 24 Cx. quinquefasciatus female mosquitoes were analyzed, representing different districts and municipalities of Jeddah Governorate. These mosquitoes were collected during routine mosquito surveillance in 2016. Collection sites for detection of target site mutations on insecticide resistance genes are shown in Fig. 1, and a map was generated using ArcGIS 10.1 ArcMap software (ESRI, Redlands, CA, USA). Taxonomic identification and ecological information on the analyzed samples are mentioned elsewhere [41].

\section{DNA extraction and target gene mutation sequencing}

For DNA extraction, individual mosquito samples, each was homogenized in a mixer mill (Jingxin, Shanghai, China) in $250 \mu \mathrm{l}$ of ATL (Qiagen, Hilden, Germany) and $20 \mu \mathrm{l}$ of proteinase K (Qiagen, Hilden, Germany) with two 3-mm steel balls were added to each tube. The homogenates were incubated at $56^{\circ} \mathrm{C}$ overnight in an oscillating thermo-block. The homogenate tubes were then centrifuged at $1000 \times \mathrm{rpm}$ for $3 \mathrm{~min}$ at room temperature. A $200 \mu \mathrm{l}$ aliquot of supernatant from each homogenate was added to the Roche ${ }^{\circledR}$ MagNA Pure 96 sample plate, and placed into to the MagNA Pure 96 System for automated DNA extraction (MagNA Pure 96 DNA and Viral NA Small Volume Kit, Roche, Basel, Switzerland) according to the manufacturer's instructions DNA was eluted in $50 \mu$ of buffer solution.

For Ae. aegypti, the primer set IIS5-6(3)F and IIS5-6(3)R were used to amplify the IIS6 domain of the VGSC, spanning the codons 989, 1011, 1016, and the introns between exons 20 and 21; while the codon 1534 domain IIIS6 was amplified by the primer set AaNa31F and AaNa31R [42]. The ace-1 gene region spanning the position 119 was amplified by the primer set Ace-1F and Ace-1R [43]. For Cx. quinquefasciatus, the VGSC region spanning the 1014 codon was amplified by the primer set Cq1 and Cq2 [44]. The fragment containing the ace-1 mutation was amplified by the primer set Moustdir and Moustrev [45].

The PCR amplification products were separated on 1.5\% agarose gel electrophoresis, purified, and sequenced in both directions by Sangon Biotech (Shanghai, China). The peak spectrum (chromatogram) of the sequencing data was determined using Bio-edit v.7.2.6 [46]. The allelic information for certain ambiguous sequences, cloning and plasmid sequencing were performed for confirmation.

\section{Phylogenetic analysis}

The obtained VGSC IIS6 fragments spanning the introns flanked by exons 20 and 21 were aligned with available sequences retrieved from GenBank using ClustalW2 [47] with default settings, which were manually adjusted when was necessary. Both intra- and inter-genotypes (type A and type B) of introns spanning exons 20 and 21 divergences were examined using the Kimura's 2-parameter (K2P) distance model [48] in MEGA v.7.0 [49]. Based on the Akaike information criterion, the best-fit model for the alignment was determined using Model test 3.7 [50], in cooperation with PAUP* v.4.0b10 [51]. Consequently, calculation of the maximum likelihood (ML) and Bayesian likelihood trees were completed under the TVM + G model for introns between exons 20 and 21 on the VGSC. Both the ML and neighbour-joining trees were constructed using MEGA v.7.0 software, with 1,000 bootstrap replicates. The Bayesian tree was built with MrBayes v3.2.6 [52] on the CIPRES portal (www.phylo.org/) [53], run for 10 million generations, with the first $25 \%$ generations discarded as burn-in. The trees were visualised using Figtree v1.4.2 (http://tree.bio.ed.ac.uk/software/figtree/). Both intra- and interspecific divergences of introns spanning between exons 20 and 21 were calculated using K2P distance model [48] in MEGA v.7.0. 


\section{Statistical analysis}

Pearson's Chi-squared test was applied to analyze associations among mutation codons and type of introns between exons 20 and 21 on VGSC. The tests were performed in SPSS v.20 (IBM Corp., Armonk, NY, USA).

\section{Results}

\section{Mapping of the target resistance-associated genes in Ae. aegypti and $\mathrm{Cx}$. quinquefasciatus}

The target resistance-associated genes successfully amplified from a total of $20 \mathrm{Ae}$. aegyptiand $21 \mathrm{CX}$. quinquefasciatus. The sequences originally obtained in this study have been deposited in the GenBank under the accession numbers MN997310-MN997399. Three non-synonymous VGSC mutation alleles, S989P, V1016G and F1534C were identified from specimens of wild Ae. aegypti. The presence of a silent mutation (TTG to TTA) at position (Leu, L) 982 (14/20) was found. No I1011M and F1552C mutations were identified. In addition, two types of introns between exons 20 and 21 , type A (250 bp) and type B (234 bp) were identified.

Six genotypes of the combination 989, 1016, and 1534 positions were documented including SVF/PGF, SVF/SVC, SVC/SVC, PGF/PGF, PGF/PGC, PGC/PGC (Table 1). Among them, PGF/PGC was the dominant genotype, detected in $45 \%$ of the examined samples, followed by the SVC/SVC genotype with $25 \%$. Genotypes of SVF/PGF, SVF/SVC, and PGC/PGC were observed less frequently. Allele frequencies are shown in Table 2. The V1016G mutation seems to be more widely distributed with high frequency $(67.50 \%)$ in Jeddah, than that of the F1534C mutation (55.00\%). Both TTG982TTA $\left(X^{2}=22.000, d f=2, P<0.001\right)$, and S989P $\left(X^{2}=44.000, d f=4, P<\right.$ $0.001)$ co-occur with the presence of V1016G. All TTG982TTA, and S989P coexist with the haplotype harboring the V1016G mutation. In individuals carrying homozygous F1534C mutation in the IIIS6 region, no coexistence of other mutations on 982, 989, and 1016 codons in IIS6 domain was observed. The type A intron between exons 20 and 21 was strongly linked to the V1016G mutation.

Table 1

Frequency (percentage) of triple-locus $k d r$ genotypes, shown as amino acid codon number 989, 1016 and 1534 in the voltage-gated sodium channel gene (VSGC) of Aedes aegypti collected from Jeddah, Saudi Arabia.

\begin{tabular}{|lllllll|}
\hline Genotype & SVF/PGF & SVF/SVC & SVC/SVC & PGF/PGF & PGF/PGC & PGC/PGC \\
\hline No. & 1 & 1 & 5 & 3 & 9 & 1 \\
\hline Percentage (\%) & 5.00 & 5.00 & 25.00 & 15.00 & 45.00 & 5.00 \\
\hline
\end{tabular}


Table 2

Frequency (percentage) of point mutations (S989P, V1016G, F1534C) and intron types identified in the voltagegated sodium channel gene (VSGC) of Aedes aegypti collected from Jeddah, Saudi Arabia.

\begin{tabular}{|c|c|c|c|c|c|c|c|c|c|c|c|}
\hline \multirow{2}{*}{$\begin{array}{l}\text { Codon } \\
\text { Allele type }\end{array}$} & \multicolumn{3}{|l|}{989} & \multicolumn{3}{|l|}{1016} & \multicolumn{3}{|l|}{1534} & \multicolumn{2}{|c|}{$\begin{array}{l}\text { Intron type } \\
\text { between } \\
\text { exons } 20 \text { and } \\
21\end{array}$} \\
\hline & $S$ & \multicolumn{2}{|c|}{$\mathrm{P}$} & V & \multicolumn{2}{|c|}{ G } & $\mathrm{F}$ & \multicolumn{2}{|c|}{ C } & $\begin{array}{l}\text { Type } \\
\text { A }\end{array}$ & $\begin{array}{l}\text { Type } \\
\text { B }\end{array}$ \\
\hline No. & 13 & \multicolumn{2}{|c|}{27} & 13 & \multicolumn{2}{|c|}{27} & 18 & \multicolumn{2}{|c|}{22} & 28 & 12 \\
\hline $\begin{array}{l}\text { Percentage } \\
(\%)\end{array}$ & 32.50 & \multicolumn{2}{|c|}{67.50} & 32.50 & \multicolumn{2}{|c|}{67.50} & 45.00 & \multicolumn{2}{|c|}{55.00} & 70.00 & 30.00 \\
\hline Genotype & SS & SP & PP & VV & VG & GG & $\mathrm{FF}$ & $\mathrm{FC}$ & $\mathrm{CC}$ & $\begin{array}{l}\text { Type } \\
\text { A }\end{array}$ & $\begin{array}{l}\text { Type } \\
\text { B }\end{array}$ \\
\hline No. & 6 & 1 & 13 & 6 & 1 & 13 & 4 & 10 & 6 & 14 & 6 \\
\hline $\begin{array}{l}\text { Percentage } \\
(\%)\end{array}$ & 30.00 & 5.00 & 65.00 & 30.00 & 5.00 & 65.00 & 20.00 & 50.00 & 30.00 & 70.00 & 30.00 \\
\hline
\end{tabular}

The haplotype with type A intron was in association with V1016G mutation $\left(X^{2}=22.00, d f=2, P<0.001\right)$ and wild-type $\mathrm{F} 1534\left(\mathrm{X}^{2}=11.75, \mathrm{df}=2, \mathrm{P}=0.003\right)$. By contrast, the $\mathrm{F} 1534 \mathrm{C}$ mutation was strongly linked with the type B intron with all of the SVC/SVC genotype linked with the type B intron. The allele type on 1016 position was highly correlated with the other two mutations, 989 and 1534, especially the 989 position $\left(\chi^{2}=44.00, d f=4\right.$, $\mathrm{P}<0.001)$. All homozygous V1016G mutations linked with homozygous S989P mutation, V1016G heterozygous mutation linked with heterozygous S989P mutation, whereas wild-type of V1016 is linked with the S989. Individuals possessing homozygous wild-type V1016 harbored homozygous (5/6) or heterozygous (1/6) of the F1534C mutation, however, no wild-type homozygous F1534 coexisted.

\section{Phylogenetic analysis of intron types identified in the voltage- gated sodium channel gene ( VSGC ) of Aedes aegypti}

The intron located between exons 20 and 21 on VGSC identified in our study and those retrieved from the GenBank were compared and used to calculate the evolution of the $k d r$ mutations. The studied 158 sequences can be clearly divided into two clades with high bootstrap values, one clade contained the sequences possessing type A intron, while the second clade contained those sequences having type B intron (Fig. 2). The intra-group genetic distances of type $A$ and type $B$ introns were 0.001 and 0.049 , respectively, whereas the intergroup was 0.355. The two intron types are detected in four continents, Africa, Asia and Latin and North America, suggesting that they were distributed paraphyletically. In Latin America (Brazil, El-Salvador, Guatemala) and North America (USA: California and Hawaii), the ratio of type A to type B intron were 5:1 and 4:0, respectively; while in Asia (China, India, Indonesia, Malaysia, Myanmar, Philippines, Saudi Arabia, Singapore, Sri Lanka, Thailand, Viet Nam), the ratio was 88:37; and in Africa (Ghana, Kenya, Malawi, Nigeria, Zambia, Zimbabwe), the ratio was 4:17. In addition, for global analysis of these data, we added specific 989, 1016 and 1534 allele types of those sequences, available from other studies. The results of analysis of the associations between the three codons and the intron types reported, showed that the allele type of 1016 position was more related to the 989 
position $\left(X^{2}=49.910, d f=4, P<0.001\right)$ than to the 1534 position $\left(X^{2}=16.297, d f=4, P=0.003\right)$. While the intron polymorphism was more correlated to the 1016 position $\left(X^{2}=34.333\right.$, $\left.d f=2, P<0.001\right)$ than the 1534 position $\left(X^{2}=18.005, d f=2, P<0.001\right)$ (Table 3).

Table 3

Chi square analysis of the linkage between point mutations and type $A$ and $B$ introns located between exons 20 and 21 on voltage-gated sodium channel gene (VGSC) reported in Aedes aegypti from Jeddah, Saudi Arabia and homologous sequences retrieved from the GenBank from other geographic regions.

\begin{tabular}{|c|c|c|c|c|c|}
\hline & & & $x^{2}$ & Df & $P$ \\
\hline \multirow[t]{5}{*}{1016} & 982 & Jeddah & 22.000 & 2 & 0.000 \\
\hline & \multirow[t]{2}{*}{989} & Jeddah & 44.000 & 4 & 0.000 \\
\hline & & Global & 49.910 & 4 & 0.000 \\
\hline & \multirow[t]{2}{*}{1534} & Jeddah & 15.402 & 4 & 0.004 \\
\hline & & Global & 16.297 & 4 & 0.003 \\
\hline \multirow[t]{4}{*}{ Intron type between exons 20 and 21} & \multirow[t]{2}{*}{1016} & Jeddah & 22.000 & 2 & 0.000 \\
\hline & & Global & 34.333 & 2 & 0.000 \\
\hline & \multirow[t]{2}{*}{1534} & Jeddah & 11.746 & 2 & 0.003 \\
\hline & & Global & 18.005 & 2 & 0.000 \\
\hline
\end{tabular}

\section{Point mutations and polymorphisms on VGSC and ace-1 in CX. quinquefasciatus}

The majority of the entire successfully sequenced partial VGSC gene with kdr mutation in Cx. quinquefasciatus was the homozygous genotype TTT/TTT (90.48\%), except two of them was the heterozygous genotype TTT/TTA.

All the detected ace-1 gene was the heterozygous genotype GGC/AGC.

\section{Discussion}

In the current study, three non-synonymous point mutations on 989, 1016 and 1534 positions and type $A$ and $B$ introns on VGSC were reported detected in field-caught Ae. aegypti mosquito samples in 2016 from Jeddah, Saudi Arabia. These mutations are commonly associated with $k d r$ worldwide due to the intensive use of pyrethroid and organophosphates insecticides in routine mosquito control operations. Chemical insecticides have been extensively used for insect disease vectors and other pests management and control in Jeddah and other mosquito-prone cities in Saudi Arabia. There are four major categories of insecticides: organochlorines, organophosphates, carbamates and pyrethroids [2,30]. The pyrethroids have been used in indoor/outdoor IRS 
since 1980s-90 s for mosquito control. The long-term pyrethroids utilization has resulted in the development of resistance to almost all classes of insecticides in major vectors of diseases including Ae. aegypti Ae. albopictus, Cx. tritaeniorhychus, Cx. quinquefasciatus, and many Anopheles species [3, 18, 21, 33, 36, 37].

No samples that harbored the wild-type at the three mutation loci simultaneously were identified. The I1011M/V mutation was absent in Jeddah, even though this substitution of isoleucine on 1011 position is prevalent in Latin America countries [39, 42, 54-56], and spread in Thailand and Vietnam but at low frequencies [57, 58]. Yet, the situation has persisted with increasing urbanization, climate changes and sustained Aedes-Culex endemicity estimated at 31-56\% prevalence rate. The mitigation and slowing down the spread and impact of insecticide resistance development in disease vector populations, needs integrated laboratory assessment coupled with surveillance, monitoring and evaluation to better understand and obtain the necessary scientific knowledgebased guidance and informing pesticides policies on selection, purchase and the long-term and sustainable vector control efforts.

So far, to our knowledge, Saudi Arabia is the place with the most variation in $k d r$ mutations associated with high resistance to pyrethroids in the Middle East countries, probably due to long-term usage of high doses of pyrethroids in vector control programs in and all major cities, especially in Jeddah since the first dengue epidemics in 1994. This is different from the reported kdr polymorphisms of Ae. aegyptipopulations from India and Pakistan. High prevalence of the F1534C mutation, and a low frequency of I1011M/V and V1016I mutations, with the absence of the 989P allele, were observed in India [25]. In Pakistan, all the studied mosquito samples harbored the homozygous F1534C mutation, whereas no mutation on the 1016 position has been identified yet [59].

The percentage of homozygous V1016G and S989P mutations co-occurrence was high (65.0\%) in Jeddah. The V1016G mutation plays a critical role in $k d r$ resistance against pyrethroid compounds $[22,23,59]$. Linkage association has been observed between the 989 and 1016 loci in Ae. aegyptipopulations of Saudi Arabia [18], Myanmar [60], Thailand [61]. The application of IRS and larvicides to reduce and control populations abundance of Aedes, Culex and Anopheles mosquitoes responsible for dengue, Rift Valley, West Nile and malaria infections witnessed significant decline in the years 1970s and 1980s, respectively. With the persistent mosquito-borne disease foci and endemicity in the major Saudi cities, Jeddah, Makkah, Madinah, Aseer and Jazan, in the SouthWestern and southern borders regions, renewed governments efforts ushered in enhanced pest surveillance coupled with integrated vector control programs implementation showed significant decline of total national cases from 2000 till now.

The occurrence of S989P was highly linked to the V1016G mutation as a compensatory mutation to reduce the fitness cost on the mosquito [22] and strengthen the response of V1016G to permethrin and deltamethrin [62]. Additionally, double homozygous mutations at positions 989 and 1016 combined with either wild-type or heterozygous mutation at 1534 position were common in Jeddah's population, in agreement with the speculation that the 1016 and 1534 loci were not independent for linkage disequilibrium analysis [63]. Further, the genotype with the triple mutation haplotype predominated in the tested mosquito samples, with an occupation of 45\% (PGC/PGF). Additionally, one individual possessing triple homozygous mutation on 989 , 1016, and 1534 loci was found in this study. In field-collected mosquitoes, the triple homozygous mutation was in low frequency in the population $[32,59,60,64,65]$, probably due to fitness cost exerted on the mosquito by this triple mutation $[62,66]$. Whereas, the artificially-introduced triple mutation, using the Xenopus oocyte 
expression systems, appeared to confer a higher level of resistance to both permethrin and deltamethrin than those carrying the individual mutations, probably for synergistic effect of the combination of mutant alleles [22, 23,62 ]. Li et al., [30] suggested that the F1534C mutation could possibly serve as a compensatory mutation for reducing the fitness cost on the mosquito induced by the V1016G mutation. Thus, whether the homozygous triple mutations on 989,1016 , and 1534 loci showing insecticide resistance in field mosquitoes is yet to be ascertained.

The intron polymorphism of VGSC could serve as a marker to study the evolution of $k d r$ mutations [38, 54]. Based on sequence size, the introns located between exons 20 and 21 were classified into type $A(250 \mathrm{pb})$ and type $B(234 \mathrm{pb})$ [54]. Analysis of intron sequences obtained in our study and homologous sequences retrieved from GenBank showed that the intron type was significantly associated with $1016\left(X^{2}=34.333, d f=2, P=0.000\right)$, and $1534\left(X^{2}=18.005, d f=2, P=0.000\right)$ allele type. Further, all the individuals possessing the V1016G mutation, either heterozygous or homozygous harbored the type $A$ intron, but not vice versa. This result is in contrast to Saavedra-Rodriguez et al.,'s [39] suggestion that the V1016G mutation was distributed independently in haplotypes possessing either the type A or type B intron. Additionally, the global data analysis showed that the two intron types were distributed evenly in both heterozygous or homozygous F1534C mutation, but the homozygous wild-type F1534 was mainly associated with the type A intron, and not vice versa as well. In Jeddah, six of the examined mosquito individuals were harboring the homozygous F1534C mutation. Among them, five individuals with the SVC/SVC genotype possessed the type B intron, while the individual carrying the homozygous triple mutation (PGC/PGC) was coupled with the type A intron. In Taiwan, China, the F1534C was found to be strictly associated with the type B intron, and the V1016G mutation coexisted with the type A intron [38]. By contrast, in Africa, the point mutation at 1534 site was found to be strongly linked with the type $A$ intron in Ghana, but only one heterozygote point mutation V1016I was recorded, except the F1534C mutations [67]. Additionally, V1016I was reported to locate at the haplotype possessing the type A intron, with no 1534 variation observed in Brazil $[54,68]$. The mutation at position 1016 is associated with resistance to both type I and II pyrethroids, while the $\mathrm{F} 1534 \mathrm{C}$ allele is primarily associated with resistance to type I pyrethroids, and the V1016I mutation did not alter channel sensitivity to pyrethroids in Xenopus oocytes [22, 23]. Based on the evidences above, we speculate that $k d r$ mutations incline to coexist with the type $\mathrm{A}$ intron. When a mutation occurred in either 1016 or 1534 positions, it would be linked to the type $A$ intron. While in case of co-mutation, the type $A$ intron distributes more frequently with the $\mathrm{V} 1016 \mathrm{G} / \mathrm{I}$ mutation rather than the mutation F1534C. It is probably due to reducing the fitness cost on the mosquito by increasing compensatory advantage or linkage disequilibrium $[62,69]$.

It is speculated that the type $B$ intron is the ancestral clade of clade 1 , because that the Ae. aegypti was originally from Africa and the majority of African individuals belong to type B [67][70]. In view of the phylogenetic tree obtained with available global data of the intron sequences, $100 \%, 83.33 \%$ and $70.40 \%$ individuals from North America, Latin America and Asian countries respectively, were type A intron. By contrast, $73.91 \%$ of sequences from Africa belonged to the type B intron. According to our analyses above, the type $A$ intron coexistence with $k d r$ mutations is common, especially the V1016G mutation; while the type B couples with the wild-type or F1534C mutation when the V1016G mutation is absent. These variable pattern of association, may reflect the history of insecticide treatment in different continents. The major disease burden of malaria is in Africa and South-East Asia [71]. The mutation on 1534 locus may have arisen in Aedes species due to earlier or current use of DDT for larvicides spraying and IRS to control malaria since the middle of the last century $[42,72]$. 
Whereas both in Latin and North America countries, vector control is mainly focused on Aedes species (mainly Ae. aegypti and Ae. albopictus) vectors of dengue, Chikungunya, yellow fever, and Zika [73]. For the domestic and endophagic habitats of $A e$. aegypti, pyrethroid insecticides are widely and randomly used in household aerosol insecticides for public and personal protection, since that pyrethroids have the advantages of high efficacy against mosquito vectors, low mammalian toxicity and short residual action [74]. In South Asia countries, the higher frequency of type $A$ intron than that of type B may indicate different selective pressures resulting in different histories of intense and unrestricted insecticide usage. It is probably that first, the F1534C mutations occurred due to the extensive use of DDT or type I pyrethroids, as both insecticides have the same target site [42], then V1016G has been selected by the subsequent and wide use of type II pyrethroids, as cypermethrin and deltamethrin [60].

Reports on target-site mutations on VGSC and ace-1 genes of Cx. quinquefasciatus are rare compared of that on Ae. aegypti. However, the insecticide resistance level of the $C x$. quinquefasciatus in Jeddah may even worse as inferred from the fixation of mutation allele both on VGSC and ace-1 genes. In the studied mosquito samples in Jeddah, the $k d r$ homozygous genotype TTT/TTT occupied most $(90.48 \%)$ of the individuals. While the heterozygous genotype GGC/AGC on ace-1 gene was detected in all samples of $C x$. quinquefasciatus. This is probably related to constant exposure due the extensive use of insecticides for routine spraying campaigns of public health, or for domestic use against mosquito vectors and other insect pests in Jeddah region. Similarity, the homozygous susceptible 1014 genotype was absent in samples collected in 2011 from Zanzibar, East Africa [75]. In India, most of the studied mosquito samples harbored the heterozygous genotype TTT/TTA, with the frequency of $75 \%$ in $C x$. quinquefasciatus samples [44].

Both the non-synonymous mutation G119S (GGC/AGC) and silent mutation T506T on ace-1 [43] were not detected in our sequences in Ae. aegypti samples from Jeddah. While it does not indicate that the Ae. aegypti population was completely susceptible to organophosphate and carbamate compounds, as both types of insecticides have the same target site. In bioassay tests, the Ae. aegypti population from Jeddah was tolerant to the fenitrothion [18], indicating that other than $k d r$ mutations, or more likely, enzymatic mechanisms are involved in conferring resistance in mosquitoes. In Central Java, Indonesia, although the G119S mutation was absent, a degree of resistance to malathion was observed in Ae. aegypti [1].

\section{Limitations of this study}

One major limitation of our study is the small sample sizes of mosquitoes used for detection on target site mutations of insecticide resistance genes. Whereas, the primary survey reveals the widespread of $k d r$ in predominant mosquitoes in Jeddah. Further, bioassay tests on both larvae and adult mosquitoes are required to evaluate the status of insecticide resistance in practice. And the underlying metabolic mechanism responsible for insecticide resistance is worth exploring.

\section{Conclusions}

The current study involved a wider geographic region and covering a larger sample of Ae. aegypti vector populations in Jeddah. The results showed that six genotypes of the three codon combinations, 989, 1016, and 1534 were spreading in Jeddah's Ae. aegypti population, with the PGF/PGC as the dominate one. Two types of introns between exon 20 and 21 on VGSC for the first time are identified in Saudi Arabia. Linkage associations 
have been shown between V1016G with S989P, V1016G with F1534, and V1016G with type A intron. High prevalence of $k d r$ mutations implies that the local $A e$. aegypti mosquitoes are pyrethroid-resistant, and the level of resistance may increase with the extensive and intensive use of pyrethroid, as the active ingredients in household insecticide and spatial repellents. To the best of our knowledge, this is the first report of resistantassociated mutations in field caught Cx. quinquefasciatus in Saudi Arabia. The fixation of L1014F allele on VGSC and G119S on ace-1 gene was detected in local Cx. quinquefasciatus populations, with the frequency of $95.24 \%$ and $100 \%$, demonstrating that traditional insecticides are likely to be losing efficacy through fogging activities or IRS.

The primary analyses showed the widespread of kdrmutation both in Ae. aegyptiand Cx. quinquefasciatus, thus threating the efficiency of vector control against mosquito-borne diseases. It raises the attention that widerange of surveillance the polymorphism and distribution of insecticide target site mutations in mosquitoes in this region. The mapping of the nation-wide distribution and polymorphism of target site mutation against insecticide resistance will provide valuable consensus for forecasting the status of insecticide resistance in mosquito populations of Jeddah and other regions of Saudi Arabia. Additionally, regular monitoring and documenting the dynamics of target mutation gene and their associations with the insecticide resistance phenotype is important in the surveillance system of preventing mosquito-borne diseases through effective vector control strategies.

Meanwhile, the above findings suggest that alternative insecticides in areas, and proper use of insect growth regulator or biocontrol approaches for integrated vector control programs should be considered in Jeddah and neighborhoods. Albeit, substantial Saudi government and Jeddah municipality commit and invest in innovative operational research, research and development on more early-detection of insecticide resistance and effective control measures and tools for discovery and utilization, including vaccines for local and pilgrims' immunization programs implementation. With the establishment of the Public Health Pest Laboratory of Jeddah Governorate, sustainable funding is imperative to provide evidence for coherent and coordinated temporal and spatial epidemiological IVM and pesticide choices implementation amongst all stakeholders. Furthermore, strengthening laboratory and epidemiologic capacity building programs for health workers, health professional, and community engagement including and private-public sectors partnerships on mosquito vector, dengue and emerging threat and epidemics sustained control towards elimination.

\section{Abbreviations}

ace-1

acetylcholinesterase 1; DENV:dengue virus; $k d r$.knockdown resistance; K2P:Kimura's 2-parameter; ML:maximum likelihood; IRS:indoor residual spraying; IVM:integrated vector management; VGSC:voltage-gated sodium channel

\section{Declarations}

\section{Ethics approval and consent to participate}

No specific permits were required for this study. The study did not involve endangered or protected species. 


\section{Consent for publication}

Not applicable.

\section{Availability of data and materials}

All data generated or analyzed during this study are included in this published article. The newly generated sequences were submitted to the GenBank database under the accession numbers MN997310-MN997399.

\section{Competing interests}

The authors declare that they have no competing interests.

\section{Funding}

The Special Foundation of Basic Science and Technology Resources Survey of Ministry of Science and Technology of China (grant no. 2017FY101200).

\section{Author contributions}

The paper was conceived Tambo Ernest, Yuan Fang, Emad I.M. Khater, Yi Zhang. The investigation was conducted by Yuan Fang, Emad I.M. Khater, Hany A. Kamal, Tambo Earnest, Data curation was performed by Tambo Ernest, Emad I.M. Khater, Hany A. Kamal, Fahd S. Alzahrani, Mohamed H. Alzahrani for mosquito collection and providing reagents and materials, Yuan Fang for conducting molecular work and phylogenetic and statistical analyses, and Jin-Bo Xue for drawing map of mosquito collection sites. Funding acquisition: Yi Zhang, Tambo Ernest, Emad Khater and XiaonNong Zhou. Yuan Fang, Tambo Ernest and Yi Zhang wrote the first draft and Yuan Fang, Tambo Ernest, Yi Zhang, Jin-Bo Xue, Emad I.M. Khater, Hany A. Kamal reviewed and edited

\section{Acknowledgments}

Not applicable.

\section{References}

1. Sayono S, Hidayati APN, Fahri S, Sumanto D, Dharmana E, Hadisaputro S, et al. Distribution of voltagegated sodium channel (Nav) alleles among the Aedes aegypti populations in Central Java Province and its association with resistance to pyrethroid insecticides. PLoS One. 2016;11:e0150577.

2. Liu H, Xie L, Cheng P, Xu J, Huang X, Wang H, et al. Trends in insecticide resistance in Culex pipiens pallens over 20 years in Shandong, China. Parasite Vector. 2019;12:167.

3. Moyes CL, Vontas J, Martins AJ, Ng LC, Koou SY, Dusfour I, et al. Contemporary status of insecticide resistance in the major Aedes vectors of arboviruses infecting humans. PLoS Neglect Trop Dis. 2017;11:e0005625.

4. Brengues C, Hawkes NJ, Chandre F, Mccarroll L, Duchon S, Guillet P, et al. Pyrethroid and DDT crossresistance in Aedes aegypti is correlated with novel mutations in the voltage-gated sodium channel gene. Med Vet Entomol. 2003;17:87-94. 
5. Liu N. Insecticide resistance in mosquitoes: impact, mechanisms, and research directions. Annu Rev Entomol. 2015;60:537-59.

6. Al-Saeed MS, El-Kafrawy SA, Farraj SA, Al-Subhi TL, Othman NA, Alsultan A, et al. Phylogenetic characterization of circulating Dengue and Alkhumra hemorrhagic fever viruses in Western Saudi Arabia and lack of evidence of Zika virus in the region: a retrospective study, 2010-2015. J Med Virol. 2017;89:1339-46.

7. Heilman JM, De Wolff J, Beards GM, Basden BJ. Dengue fever: a Wikipedia clinical review. Open Med. 2014;8:e105-15.

8. Organji SR, Abulreesh HH, Osman GE. Circulation of dengue virus serotypes in the city of Makkah, Saudi Arabia, as determined by reverse transcription polymerase chain reaction. Can J Infect Dis Med Microbiol. 2017;2017:1646701.

9. Mohammed MS, Abakar AD, Nour BY, Dafalla OM. Molecular surveillance of dengue infections in Sabya governate of Jazan region, Southwestern Saudi Arabia. Int J Mosq Res. 2018;5:125-32.

10. The Ministry of Health of Saudi Arabia. The Statistical Yearbook, 2018G. 201904/1439-03/40H.

11. Alhaeli A, Bahkali S, Ali A, Househ MS, El-Metwally AA. The epidemiology of Dengue fever in Saudi Arabia: a systematic review. J Infect Public Health. 2016;9:117-24.

12. Ashshi A. The prevalence of dengue virus serotypes in asymptomatic blood donors reveals the emergence of serotype 4 in Saudi Arabia. Virol J. 2017;14:107.

13. Alikhan M, Al Ghamdi K, Mahyoub JA. Aedes mosquito species in western Saudi Arabia. J Insect Sci. 2014;14:69.

14. Khater EIM, Sowilem MM, Sallam MF, Alahmed AM. Ecology and habitat characterization of mosquitoes in Saudi Arabia. Trop Biomed. 2013;30:409-27.

15. Khan E, Barr KL, Farooqi J, Prakoso D, Abbas A, Khan ZY, et al. Human west nile virus disease outbreak in Pakistan, 2015-2016. Front Public Health. 2018;6:20.

16. Balakrishnan A, Thekkekare R, Sapkal G, Tandale B. Seroprevalence of Japanese encephalitis virus \& West Nile virus in Alappuzha district, Kerala. Indian J Med Res. 2017;146:70-6.

17. Sayedahmed M. Incidence history of west nile virus in Africa and Middle East, with an emphasis on Egypt: a review. J Dairy Vet Anim Res. 2016;3:00080.

18. Nazawi AMA, Aqili J, Alzahrani M, Mccall PJ, Weetman D. Combined target site ( $k d r)$ mutations play a primary role in highly pyrethroid resistant phenotypes of Aedes aegypti from Saudi Arabia. Parasite Vector. 2017;10:161.

19. Alsheikh AA, Mohammed WS, Noureldin EM, Daffalla OM, Shrwani KJ, Hobani YA, et al. Resistance status of Aedes aegypti to insecticides in the Jazan region of Saudi Arabia. Biosci Biotech Res Asia. 2016;13:155-62.

20. Amin AM. Preliminary investigation of the mechanisms of DDT and pyrethroid resistance in Culex quinquefasciatus Say (Diptera: Culicidae) from Saudi Arabia. Bull ent Res. 1989;79:361-6.

21. Donnelly MJ, Corbel V, Weetman D, Wilding CS, Williamson MS. Black IV WC. Does kdr genotype predict insecticide-resistance phenotype in mosquitoes? Cell. 2009;25:213-9.

22. Du Y, Nomura Y, Zhorov B, Dong K. Sodium channel mutations and pyrethroid resistance in Aedes aegypti. Insects. 2016;7:60. 
23. Du Y, Nomura Y, Satar G, Hu Z, Nauen R, He S, et al. Molecular evidence for dual pyrethroid-receptor sites on a mosquito sodium channel. P Natl Acad Sci USA. 2013;110:11785-90.

24. Chang C, Shen W-K, Wang T-T, Lin Y-H, Hsu E-L, Dai S-M. A novel amino acid substitution in a voltage-gated sodium channel is associated with knockdown resistance to permethrin in Aedes aegypti. Insect Biochem Molec. 2009;39:272-8.

25. Kushwah RBS, Dykes CL, Kapoor N, Adak T, Singh OP. Pyrethroid-resistance and presence of two knockdown resistance $(k d r)$ mutations, F1534C and a novel mutation T1520I, in Indian Aedes aegypti. PLoS Neglect Trop Dis. 2015;9:e3332.

26. Yanola J, Somboon P, Walton C, Nachaiwieng W, Prapanthadara L. A novel F1552/C1552 point mutation in the Aedes aegyptivoltage-gated sodium channel gene associated with permethrin resistance. Pestic Biochem Phys. 2010;96:127-31.

27. Dusfour I, Zorrilla P, Guidez A, Issaly J, Girod R, Guillaumot L, et al. Deltamethrin resistance mechanisms in Aedes aegypti populations from three French overseas territories worldwide. PLoS Negl Trop Dis. 2015;9:117.

28. Harris AF, Rajatileka S, Ranson H. Pyrethroid resistance in Aedes aegypti from Grand Cayman. Am J Trop Med Hyg. 2010;83:277-84.

29. Kawada H, Higa Y, Futami K, Muranami Y, Kawashima E, Osei JHN, et al. Discovery of point mutations in the voltage-gated sodium channel from African Aedes aegypti populations: potential phylogenetic reasons for gene introgression. PLoS Negl Trop Dis. 2016;10:e0004780.

30. Li C, Kaufman PE, Xue R, Zhao M, Wang G, Yan T, et al. Relationship between insecticide resistance and $k d r$ mutations in the dengue vector Aedes aegypti in Southern China. Parasite Vector. 2015;8:325.

31. Du Y, Nomura Y, Zhorov BS, Dong K. Sodium channel mutations and pyrethroid resistance in Aedes aegypti. Insects. 2016;7:60.

32. Dusfour I, Zorrilla P, Guidez A, Issaly J, Girod R, Guillaumot L, et al. Deltamethrin resistance mechanisms in Aedes aegypti populations from three French overseas territories worldwide. PLoS Neglect Trop Dis. 2015;9:e0004226.

33. Li T, Zhang L, Reid WR, Xu Q, Dong K, Liu N. Multiple mutations and mutation combinations in the sodium channel of permethrin resistant mosquitoes, Culex quinquefasciatus. Sci Rep. 2012;2:781.

34. Komagata O, Kasai S, Obara I, Motoyama N, Tanaka I, Kobaysshi M, et al. Concomitant identification of subspecies and insecticide resistance-associated mutations in the mosquito Culex pipiens complex by primer extension-based genotyping. Med Entomol Zool. 2008;59:33-46.

35. Xu Q, Zhang L, Li T, Zhang L, He L, Dong K, et al. Evolutionary adaptation of the amino acid and codon usage of the mosquito sodium channel following insecticide selection in the field mosquitoes. PLoS One. 2012;7:e47609.

36. Weill M, Lutfalla G, Mogensen KE, Chandre F, Berthomieu A, Berticat C, et al. Insecticide resistance in mosquito vectors. Nature. 2003;423:136-7.

37. Scott JG, Yoshimizu MH, Kasai S. Pyrethroid resistance in Culex pipiens mosquitoes. Pestic Biochem Phys. 2015;120:68-76.

38. Chung H-H, Cheng IC, Chen Y-C, Lin C, Tomita T, Teng H-J. Voltage-gated sodium channel intron polymorphism and four mutations comprise six haplotypes in an Aedes aegypti population in Taiwan. PLoS 
Neglect Trop Dis. 2019;13:e0007291.

39. Saavedra-Rodriguez K, Urdaneta-Marquez L, Rajatileka S, Moulton M, Flores A, Fernandez Salas I, et al. A mutation in the voltage-gated sodium channel gene associated with pyrethroid resistance in Latin American Aedes aegypti. Insect Mol Biol. 2007;16:785-98.

40. Wuliandari JR, Lee SF, White VL, Tantowijoyo W, Hoffmann AA, Endersbyharshman NM. Association between three mutations, F1565C, V1023G and S996P, in the voltage-sensitive sodium channel gene and knockdown resistance in Aedes aegypti from Yogyakarta, Indonesia. Insects. 2015;6:658-85.

41. Fang Y, Ernest T, Zhang Y, Kamal HA, Alzahrani FS, Alzahrani M, et al. Molecular analysis of targeted insecticide resistance gene mutations in field Aedes aegypti and Culex quinquefasciatus mosquito vectors of arboviruses from Saudi Arabia. PLoS Neglect Trop D. 2020;In revision.

42. Harris AF, Rajatileka S, Ranson H. Pyrethroid resistance in Aedes aegypti from Grand Cayman. Am J Trop Med Hyg. 2010;83:277-84.

43. Hasmiwati H, Rusjdi SR, Nofita E. Detection of Ace-1 gene with insecticides resistance in Aedes aegypti populations from DHF-endemic areas in Padang, Indonesia. Biodiversitas. 2018;19:31-6.

44. Sarkar M, Borkotoki A, Baruah I, Bhattacharyya IK, Srivastava RB. Molecular analysis of knock down resistance $(k d r)$ mutation and distribution of $k d r$ genotypes in a wild population of Culex quinquefasciatus from India. Trop Med Int Health. 2009;14:1097-104.

45. Huchard E, Martinez M, Alout H, Douzery EJP, Lutfalla G, Berthomieu A, et al. Acetylcholinesterase genes within the Diptera: takeover and loss in true flies. Proc Biol Sci. 2006;273:2595-604.

46. Hall TA. BioEdit: a user-friendly biological sequence alignment editor and analysis program for Windows 95/98/NT. Nucleic Acids Symp Ser. 1999;41:95-8.

47. Larkin MA, Blackshields G, Brown NP, Chenna R, McGettigan PA, McWilliam H, et al. Clustal W and Clustal X version 2.0. Bioinformatics. 2007;23:2947-8.

48. Kimura M. A simple method for estimating evolutionary rates of base substitutions through comparative studies of nucleotide sequences. J Mol Evol. 1980;16:111-20.

49. Kumar S, Stecher G, Tamura K. MEGA7: molecular evolutionary genetics analysis version 7.0 for bigger datasets. Mol Biol Evol. 2016;33:1870-4.

50. Posada D, Crandall KA. MODELTEST: testing the model of DNA substitution. Bioinformatics. 1998;14:8178.

51. Wilgenbusch JC, Swofford D. Inferring evolutionary trees with PAUP*. Current Protocols in Bioinformatics. 2003;6:6.4.

52. Ronquist F, Teslenko M, van der Mark P, Ayres DL, Darling A, Hohna S, et al. MrBayes 3.2: efficient Bayesian phylogenetic inference and model choice across a large model space. Syst Biol. 2012;61:539-42.

53. Miller MA, Pfeiffer W, Schwartz T: Creating the CIPRES Science Gateway for inference of large phylogenetic trees. In: Proceedings of the gateway computing environments workshop (GCE). New Orleans: Louisiana; 2010: 1-8.

54. Martins A, Lins R, Linss J, Peixoto A, Valle D. Voltage-gated sodium channel polymorphism and metabolic resistance in pyrethroid-resistant Aedes aegyptifrom Brazil. Am J Trop Med Hyg. 2009;81:108-15.

55. Siller Q, Ponce G, Lozano S, Flores AE. Update on the frequency of lle1016 mutation in voltage-gated sodium channel gene of Aedes aegypti In Mexico. J Am Mosquito Contr. 2011;27:357-62. 
56. Martins AJ, Lima JBP, Peixoto AA, Valle D. Frequency of Val1016lle mutation in the voltage-gated sodium channel gene of Aedes aegypti Brazilian populations. Trop Med Int Healt. 2009;14:1351-5.

57. Rajatileka S, Black WC, Saavedrarodriguez K, Trongtokit Y, Apiwathnasorn C, Mccall PJ, et al. Development and application of a simple colorimetric assay reveals widespread distribution of sodium channel mutations in Thai populations of Aedes aegypti. Acta Trop. 2008;108:54-7.

58. Bingham G, Strode C, Tran L, Khoa P, Jamet H. Can piperonyl butoxide enhance the efficacy of pyrethroids against pyrethroid-resistant Aedes aegypti? Trop Med Int Healt. 2011;16:492-500.

59. Stenhouse SA, Plernsub S, Yanola J, Lumjuan N, Dantrakool A, Choochote W, et al. Detection of the V1016G mutation in the voltage-gated sodium channel gene of Aedes aegypti (Diptera: Culicidae) by allele-specific PCR assay, and its distribution and effect on deltamethrin resistance in Thailand. Parasite Vector. 2013;6:253.

60. Kawada H, Oo SZM, Thaung S, Kawashima E, Maung YNM, Thu HM, et al. Co-occurrence of point mutations in the voltage-gated sodium channel of pyrethroid-resistant Aedes aegyptipopulations in Myanmar. PLoS Neglect Trop Dis. 2014;8:e3032.

61. Srisawat R, Komalamisra N, Eshita Y, Zheng M, Ono K, Itoh T, et al. Point mutations in domain II of the voltage-gated sodium channel gene in deltamethrin-resistant Aedes aegypti (Diptera: Culicidae). Appl Entomol Zool. 2010;45:275-82.

62. Hirata K, Komagata O, Itokawa K, Yamamoto A, Tomita T, Kasai S. A single crossing-over event in voltagesensitive $\mathrm{Na}^{+}$channel genes may cause critical failure of Dengue mosquito control by insecticides. PLoS Neglect Trop Dis. 2014;8:e3085.

63. Vera-Maloof FZ, Saavedrarodriguez K, Elizondoquiroga A, Lozanofuentes S, Black WC. Coevolution of the Ile1,016 and Cys1,534 mutations in the voltage gated sodium channel gene of Aedes aegypti in Mexico. PLoS Neglect Trop Dis. 2015;9:e0004263.

64. Yanola J, Somboon P, Walton C, Nachaiwieng W, Somwang P, Prapanthadara LA. High-throughput assays for detection of the F1534C mutation in the voltage-gated sodium channel gene in permethrin-resistant Aedes aegypti and the distribution of this mutation throughout Thailand. Trop Med Int Health. 2011;16:501-9.

65. Marcombe S, Fustec B, Cattel J, Chonephetsarath S, Thammavong P, Phommavanh N, et al. Distribution of insecticide resistance and mechanisms involved in the arbovirus vector Aedes aegypti in Laos and implication for vector control. PLoS Neglect Trop Dis. 2019;13:e0007852.

66. Brito LP, Linss J, Limacamara TN, Belinato TA, Peixoto AA, Lima JBP, et al. Assessing the effects of Aedes aegypti kdr mutations on pyrethroid resistance and its fitness cost. PLoS One. 2013;8:e60878.

67. Kawada H, Higa Y, Futami K, Muranami Y, Kawashima E, Osei J, et al. Discovery of point mutations in the voltage-gated sodium channel from African Aedes aegypti populations: potential phylogenetic reasons for gene introgression. PLoS Neglect Trop Dis. 2016;10:e0004780.

68. Martins AJ, Brito LP, Linss J, Rivas GBS, Machado R, Bruno RV, et al. Evidence for gene duplication in the voltage-gated sodium channel gene of Aedes aegypti. Evol Med Public Health. 2013;2013(1):148-60.

69. Lynch M. Intron evolution as a population-genetic process. P Natl Acad Sci USA. 2002;99:6118-23.

70. Gloriasoria A, Ayala D, Bheecarry A, Calderonarguedas O, Chadee DD, Chiappero MB, et al. Global genetic diversity of Aedes aegypti. Mol Ecol. 2016;25:5377-95. 
71. WHO. World malaria report 2019. Geneva: World Health Orgnization; 2019.

72. Balkew M, Ibrahim M, Koekemoer LL, Brooke BD, Engers H, Aseffa A, et al. Insecticide resistance in Anopheles arabiensis (Diptera: Culicidae) from villages in central, northern and south west Ethiopia and detection of $k d r$ mutation. Parasit Vectors. 2010;3:40.

73. Weaver SC, Charlier C, Vasilakis N, Lecuit M. Zika, chikungunya, and other emerging vector-borne viral diseases. Annu Rev Med. 2018;69:395-408.

74. WHO. Test procedures for insecticide resistance monitoring in malaria vector mosquitoes. Geneva: World Health Orgnization; 2013.

75. Jones CM, Machin C, Mohammed K, Majambere S, Ali AS, Khatib BO, et al. Insecticide resistance in Culex quinquefasciatus from Zanzibar: implications for vector control programmes. Parasit Vectors. 2012;5:78.

\section{Figures}




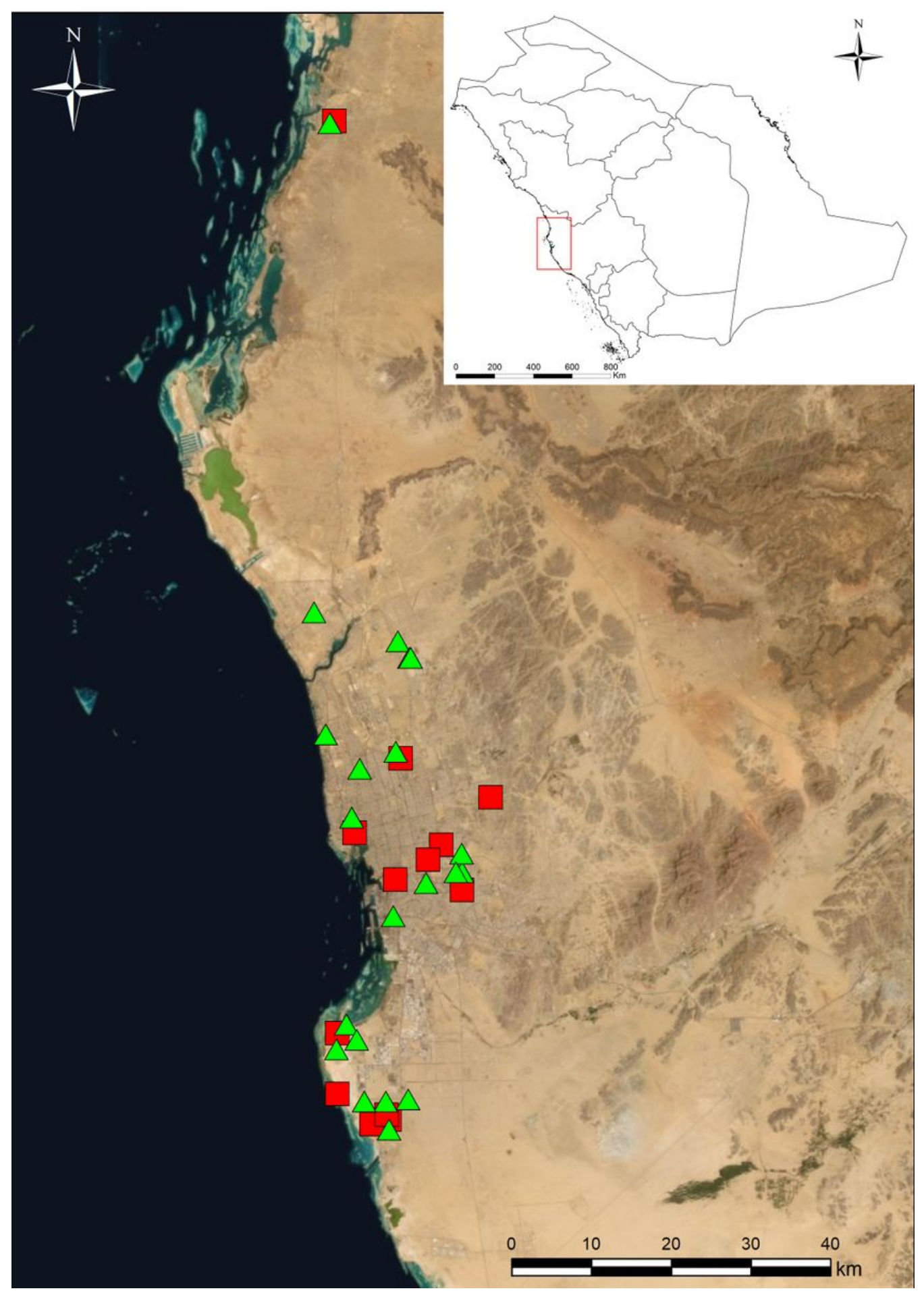

Figure 1

of mosquito collection sites for detection of target site mutations on insecticide resistance genes in 2016 in Jeddah, Saudi Arabia. Squares represent collection sites of Aedes aegypti, triangles represent sites of Culex quinquefasciatus. 


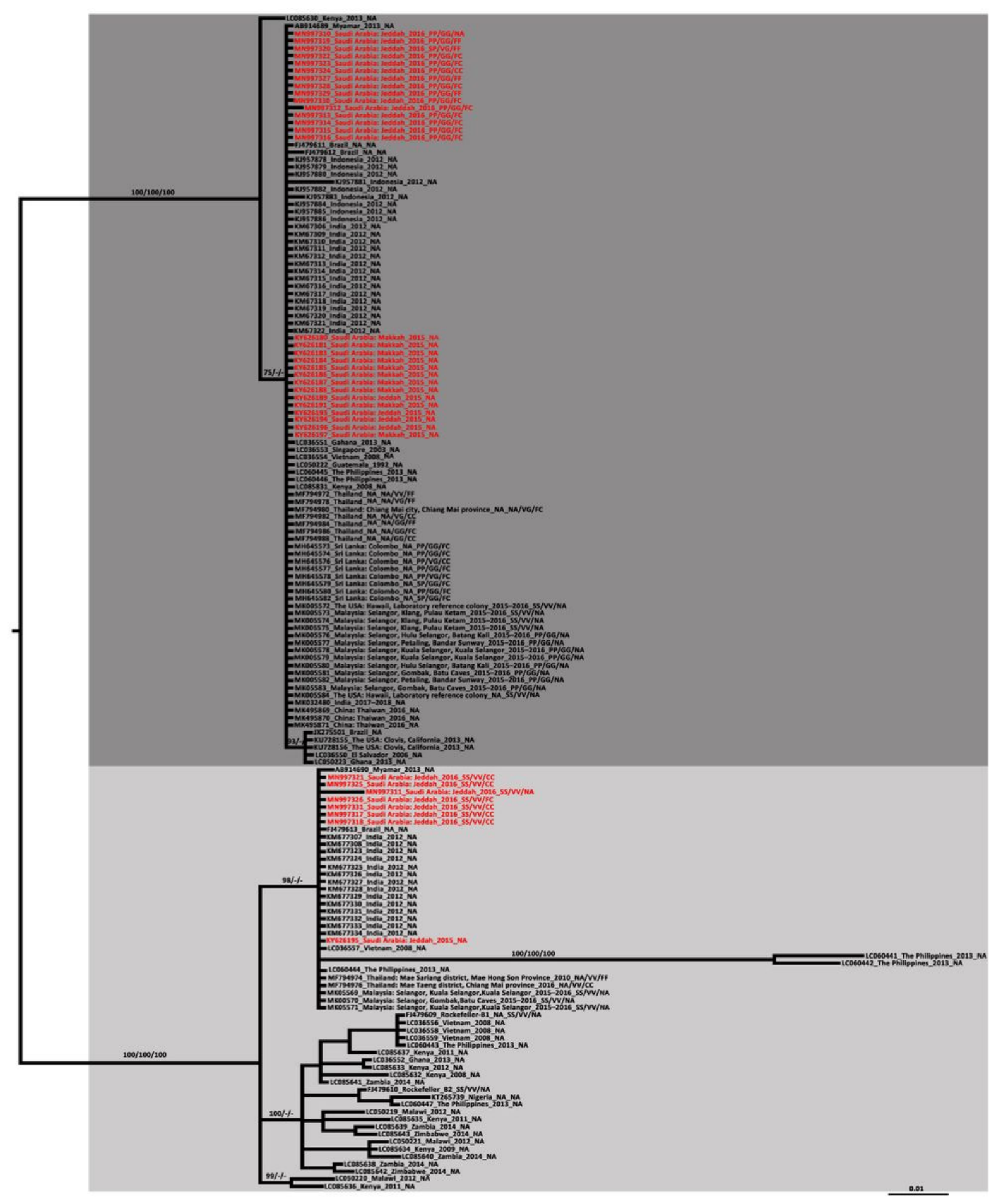

Figure 2

Maximum likelihood phylogeny of introns between exons 20 and 21 of the voltage-gated sodium channel gene of Aedes aegypti, constructed by 158 sequences under the TVM+ G model. The GenBank accession number, collection country/province and year of each sequence are noted. The sequences obtained in this study are marked in red. The numbers above the branch represent the bootstrap support of the maximum likelihood, neighbor-joining, and Bayesian analyses, respectively, based on 1000 replicates. The scale-bar indicates 0.01 nucleotide substitutions per site. Dark grey and light grey indicate the type A and B of the introns located between exons 20 and 21. 


\section{Supplementary Files}

This is a list of supplementary files associated with this preprint. Click to download.

- Presentation1.jpg 